\title{
A importância do acompanhamento farmacoterapêutico a idosos pertencentes ao grupo da "melhor idade" da FACESA
}

\author{
Nathana Lima de Sá1 Renata Costa Fortes ${ }^{1}$
}

\begin{abstract}
RESUMO
Objetivo - Investigar a importância do acompanhamento farmacoterapêutico a idosos. Métodos - Estudo transversal descritivo realizado na Faculdade de Ciências e Educação Sena Aires de Valparaíso de Goiás em setembro de 2012. Amostra de 30 idosos, de ambos os sexos, participantes do grupo da "Melhor Idade". Utilizou-se questionário adaptado do Método Dáder de Acompanhamento Farmacoterapêutico, questionário SF 36, variáveis socioeconômicas e sociodemográficas, hábitos de vida, estado de saúde, uso de medicamentos e autopercepção da qualidade de vida. Resultados - Observou-se que $56,7 \%(n=17)$ dos idosos utilizavam medicamentos por conta própria, 63,3\% ( $n=19)$ faziam uso de chás ou garrafadas, $76,7 \%(n=23)$ de polifarmácia e 10\% $(n=3)$ eram alérgicos a algum medicamento. Não houve interação medicamentosa em $56,7 \%(n=17)$ dos casos e 13,3\% (n=4) tiveram risco potencial de interações medicamentosas. Conclusão - Os resultados apontam a necessidade de um acompanhamento farmacoterapêutico de qualidade para os idosos no intuito de minimizar o risco potencial de interações medicamentosas entre alguns medicamentos.
\end{abstract}

Descritores: Idoso, Acompanhamento farmacoterapêutico, Qualidade de vida.

\section{The importance of the pharmacotherapeutic monitoring of elderly belonging to group of "best age" of FACESA}

\begin{abstract}
Objective - To investigate the importance of pharmacotherapeutic monitoring the elderly. Methods - Cross-sectional study conducted at the Faculty of Science and Education Sena Aires to Valparaiso de Goiás in September 2012. Sample of 30 elderly, both sexes, participants of the group "Golden Age". The questionnaire was adapted from the method Dáder Monitoring Pharmacotherapy, SF 36, socioeconomic and sociodemographic variables, lifestyle habits, health status, medication use and self-perceived quality of life. Results - It was observed that $56.7 \%$ $(n=17)$ of older people using drugs on their own, $63.3 \%(n=19)$ were using potions or teas, $76.7 \%(n=23)$ of polypharmacy and $10 \%(n=3)$ were allergic to any medicine. No drug interaction was observed in $56.7 \%(n=17)$ of cases and $13.3 \%(n=4)$ had potential risk of drugs interations. Conclusion - The results indicate the need for a pharmacotherapeutic monitoring quality for the elderly in order to minimize potential risk of drugs interations between some medications.
\end{abstract}

Descriptors: Elderly, Pharmacotherapeutic monitoring, Quality of life. 


\section{Introdução}

No século XX, sobretudo a partir dos anos 60 , o Brasil e o mundo têm sofrido uma mudança demográfica com aumento do número de idosos e, por conseguinte no perfil de morbimortalidade e de uso dos serviços de saúde ${ }^{1}$.

A transição demográfica foi acompanhada por uma mudança no perfil saúde-doença da população, com prevalência das doenças crônico-degenerativas e uso contínuo de medicamentos para tratá-las ${ }^{1}$.

Os idosos fazem uso de vários tipos de medicamentos, o que os tornam vulneráveis à riscos de Problemas Relacionados aos Medicamentos - PRMs. A atuação do farmacêutico é importante para garantir adesão ao esquema terapêutico e segurança ao usuário por meio de orientações relacionadas à administração e à promoção do uso racional de medicamentos ${ }^{2}$.

O acompanhamento farmacoterapêutico é considerado um modelo de prática inserido dentro da Atenção Farmacêutica, onde esta é utilizada para diminuir internações ou tempo de permanência em hospitais, constituindo uma intervenção terapêutica mais custo efetiva; ou seja, aquela que apresenta maior benefício e melhor custo incremental ${ }^{3}$.

Nesse novo modelo de prática farmacêutica, inicialmente, o profissional traça o perfil do paciente e procura conhecer as medicações utilizadas, as dificuldades que acompanham a farmacoterapia, as interações medicamentosas e os problemas relacionados a medicamentos presentes ${ }^{4}$.

O acompanhamento farmacoterapêutico preconiza um atendimento mais humanizado, incluindo maior tempo de atenção ao paciente a fim de investigar a posologia, aspectos relacionados ao acesso, à adesão, à administração e ao armazenamento de medicamentos; identificar reações adversas e dificuldades que o paciente apresente para seguir a prescrição médica. Além disso, ajuda no monitoramento de parâmetros bioquímicos e fisiológicos como pressão arterial, glicemia e controle do peso ${ }^{5}$.

O método Dáder foi criado com o intuito de contribuir para melhorar a efetividade e minimizar os problemas com 0 tratamento farmacológico, principalmente para aquelas pessoas que fazem uso de dois ou mais medicamentos, sendo este um modelo de atenção farmacêutica a ser seguido para auxiliar o farmacêutico a ter um padrão de acompanhamentos, diálogo e consulta ao paciente que, por sua vez, pode colocar todas as queixas e satisfações, traçando assim seu perfil farmacológico ${ }^{4}$.

Este acompanhamento é um procedimento operacional de atenção farmacêutica que almeja sempre a segurança e a qualidade de vida do paciente, buscando a linguagem interprofissional entre médico e farmacêutico para se ter uma intervenção favorável a este paciente ${ }^{6}$.

Há resultados positivos do acompanhamento farmacoterapêutico a pacientes hipertensos e diabéticos, oncohematológicos e com distúrbio bipolar, trazendo uma melhor qualidade de vida aos usuários de medicamentos por diminuir a incidência de erros associados à medicação $0^{7}$.

O objetivo do presente estudo foi investigar a importância do acompanhamento farmacoterapêutico a idosos pertencentes ao grupo da "Melhor Idade" da Faculdade de Ciências e Educação Sena Aires (FACESA) de Valparaíso de Goiás-GO.

\section{Métodos}

\section{Delineamento do estudo}

Trata-se de um estudo transversal descritivo realizado na Faculdade de Ciências e Educação Sena Aires (FACESA) de Valparaíso de Goiás - GO no mês de setembro de 2012.

\section{Casuística}

A amostra foi constituída de 30 idosos, de ambos os sexos, participantes do grupo da "Melhor Idade" da FACESA. Os critérios de inclusão utilizados na pesquisa foram indivíduos que estavam em uso contínuo de pelo menos um medicamento, capazes de responder à entrevista e aptos a receber orientações dos pesquisadores. Foram excluídos os indivíduos com idade inferior a 60 anos, considerados sem discernimento, com demência, deficientes visuais e auditivos. 


\section{Coleta de dados}

Os dados foram coletados por acadêmicos do Curso de Farmácia da Universidade Paulista, Campus Brasília - DF por meio do questionário do Método Dáder de Acompanhamento Farmacoterapêutico ${ }^{8}$ validado por Oliveira e Novaes ${ }^{9} \mathrm{e}$ adaptado para o presente estudo, do questionário SF-36 versão em português ${ }^{10}$, o questionário de Avaliação do Estado Afetivo (Humor) - Escala de Depressão Geriátrica Reduzida ${ }^{4}$ validado por Oliveira e Novaes ${ }^{9}$, e o questionário de Escala de Atividades Instrumentais de Vida Diária validado por Oliveira e Novaes ${ }^{9}$, referentes à qualidade de vida. Os dados coletados foram complementados com análise na última prescrição apresentada pelo entrevistado.

Além desses instrumentos, foram coletadas as seguintes variáveis: socioeconômicas e sociodemográficas (idade, sexo e renda familiar); hábitos de vida (tabagismo, etilismo e atividade física); estado de saúde e doenças prevalentes; queixas de saúde; medicamentos utilizados (avaliação qualiquantitativa); e autopercepção da qualidade de vida.

\section{Grupo da "Melhor Idade"}

O grupo da "Melhor Idade" faz parte do programa de extensão da FACESA. Atua em parceria com a Secretaria da Promoção Social do Município de Valparaíso de Goiás sendo articulado com a disciplina de Enfermagem em Saúde do Idoso, desenvolvendo atividades de promoção à saúde (verificação de peso, estatura, glicemia capilar, pressão arterial, massagens terapêuticas e relaxantes, atividades lúdicas e consultas multiprofissionais). Tem o intuito de possibilitar atendimento à comunidade idosa, de ambos os sexos, de Valparaíso de Goiás ${ }^{11}$.

\section{Análise estatística}

A análise dos dados foi realizada de forma descritiva com cálculo de média e desvio padrão para as variáveis quantitativas e porcentagens para as variáveis qualitativas, utilizando-se o software Microsoft Office Excel ${ }^{\circledR} 2007$.

\section{Aspectos éticos}

Este estudo foi aprovado pelo Comitê de Ética em Pesquisa em Seres Humanos da Universidade Paulista, Campus Indianópolis, São Paulo (CEP/UNIP, Indianópolis-SP) no dia 08/11/2012 sob o parecer número 142.415 e Certificado de Apresentação para Apreciação Ética (CAAE) número 08560412.5.0000.5512.

Todos os idosos que atenderam aos critérios de inclusão e que aceitaram participar da pesquisa por meio da assinatura do Termo de Consentimento Livre e Esclarecido (TCLE) após informações detalhadas sobre os objetivos e procedimentos do estudo.

Foram garantidos aos sujeitos de pesquisa o sigilo e o anonimato de suas informações, assegurando a confidencialidade e a privacidade, conforme a Resolução n 466 de 12 de dezembro de 2012 do Conselho Nacional de Saúde do Ministério da Saúde (CNS/MS).

\section{Resultados e discussão}

A amostra foi constituída de 30 idosos, sendo 86,7\% ( $n=26$ ) do sexo feminino, com média de idade de 65,4 $\pm 6,4$ anos e média de renda de $R \$ 1.106,40 \pm 855,82$ reais. Destes, $26,7 \%(n=8)$ eram solteiros, $33,3 \%(n=10)$ casados, $10 \%(n=3)$ separados ou divorciados e $30 \%(n=9)$ viúvos (Tabela 1). Esses dados corroboram com a literatura, visto que as pessoas que mais se preocupam com a saúde são as do sexo feminino, sendo estas as que têm maior perspectiva de vida².

Observou-se que apenas 10\% (n=3) dos idosos eram fumantes e 46,6\% ( $n=14)$ faziam uso de bebidas alcoólicas (Tabela 1). Evidências científicas comprovam a existência de interação medicamentosa relacionadas à ingestão de álcool ${ }^{12}$. Porém, no presente estudo essa interação não foi investigada.

Os pacientes que praticavam atividade física toda semana representaram 93,3\% ( $n=28)$ dos casos e, 86,7\% $(n=26)$ relataram considerar seu estado de saúde como bom (Tabela 1). Estudos apontam que a prática de atividade física pode influenciar significativamente na melhoria da saúde dos indivíduos, com consequente redução das consultas médicas e das filas em hospitais ${ }^{13}$. Isto pôde ser observado nesta pesquisa, uma vez que alguns idosos relataram que após terem aderido à prática de atividade física, sentiram melhora da saúde e disposição para participarem de atividades sociais.

Dos idosos analisados, $76,7 \%(n=23)$ faziam uso de mais de um medicamento ao dia (Tabela 1). A polimedicação está relacionada a interações medicamentosas e efeitos adversos como tontura e câimbras, tornando-se imprescindível uma adequada intervenção farmacêutica com o intuito de evitar ou minimizar esses efeitos ${ }^{10}$. 
As doenças relatadas com mais frequência foram: hipertensão arterial 46,7\% (n=14), artrose e insônia $20 \%$ $(n=6)$, rinite alérgica e osteoporose $20 \%(n=6)$ e gastrite e hipercolesterolemia $13,3 \%(n=4)$ (Tabela 1). Todas essas doenças implicam na utilização de diversos tipos de medicamentos que podem sofrer interações droga-droga e droga-nutriente, com impacto negativo sobre a saúde do idoso, sendo fundamental a atuação do farmacêutico ${ }^{7}$.

Os medicamentos mais utilizados pelos idosos foram os antihipertensivos e diuréticos $90 \%$ ( $n=27)$ e os antidislipidêmicos 10\% (n=3) (Tabela 1). Como esses medicamentos são de uso crônico, qualquer medicação adicional que este paciente venha utilizar, deverá haver uma atenção especial devido à associação com os medicamentos de uso contínuo ${ }^{7}$.

Tabela 1 - Características dos idosos do grupo da "Melhor Idade" da Faculdade de Ciências e Educação Sena Aires de Valparaíso de Goiás-GO. Setembro/2012 (n=30).

\begin{tabular}{|c|c|c|}
\hline Variáveis & $\mathrm{n}$ & $f \% p$ \\
\hline \multicolumn{3}{|l|}{ Estado civil } \\
\hline Solteiro & 08 & 26,7 \\
\hline Casado & 03 & 33,3 \\
\hline Separado/Divorciado & 10 & 10,0 \\
\hline Viúvo & 09 & 30,0 \\
\hline \multicolumn{3}{|l|}{ Idade (anos) } \\
\hline $60-70$ & 21 & 70,0 \\
\hline $71-80$ & 09 & 30,0 \\
\hline \multicolumn{3}{|l|}{ Sexo } \\
\hline Masculino & 04 & 13,3 \\
\hline Feminino & 26 & 86,7 \\
\hline \multicolumn{3}{|l|}{ Renda mensal (salários mínimos) } \\
\hline$\leq 1$ & 02 & 06,7 \\
\hline $1-2$ & 20 & 66,7 \\
\hline $3-4$ & 05 & 16,7 \\
\hline$\geq 4$ & 03 & 09,9 \\
\hline \multicolumn{3}{|l|}{ Tabagismo } \\
\hline Sim & 03 & 10,0 \\
\hline Não & 21 & 70,0 \\
\hline Ex-fumante & 06 & 20,0 \\
\hline \multicolumn{3}{|l|}{ Etilismo } \\
\hline Sim & 02 & 06,7 \\
\hline Não & 12 & 40 \\
\hline Raramente & 16 & 53,3 \\
\hline \multicolumn{3}{|l|}{ Prática de atividade física } \\
\hline Sim & 28 & 93,3 \\
\hline Não & 02 & 06,7 \\
\hline \multicolumn{3}{|l|}{ Estado geral de saúde } \\
\hline Bom & 26 & 86,7 \\
\hline Ruim & 04 & 13,3 \\
\hline \multicolumn{3}{|l|}{ Consumo de Medicamento } \\
\hline Sem polimedicação & 07 & 23,3 \\
\hline Com polimedicação & 23 & 76,7 \\
\hline \multicolumn{3}{|l|}{ Doenças relatadas } \\
\hline Hipertensão & 14 & 46,7 \\
\hline Artrose e insônia & 06 & 20,0 \\
\hline Rinite Alérgica e Osteoporose & 05 & 16,7 \\
\hline Gastrite e Hipercolesterolemia & 04 & 13,3 \\
\hline Bronquite & 01 & 03,3 \\
\hline
\end{tabular}




\begin{tabular}{c|c|c}
\hline Queixas de saúde & & \\
\hline Dor nas pernas & 14 & 46,7 \\
\hline Falta de memória & 14 & 46,7 \\
\hline Dor nas costas & 13 & 43,3 \\
\hline Azia & 05 & 16,7 \\
\hline Tipos de medicamentos & & \\
utilizados & & 33,8 \\
\hline Antihipertensivo & 27 & 11,3 \\
\hline Diurético & 09 & 06,3 \\
\hline Antidislipidêmico & 05 &
\end{tabular}

Legenda: $n=$ número de idosos. f\% $\mathrm{p}=$ Frequência percentual

Em relação à automedicação, constatou-se que $56,7 \%(n=17)$ dos idosos tomavam medicamentos por conta própria, $63,3 \%(n=19)$ consumiam chá ou garrafada e 10\% $(n=3)$ eram alérgicos a algum tipo de medicamento (Tabela 2). Esses fatores estão relacionados a interações e intoxicações, principalmente em idosos que representam um dos grupos mais vulneráveis, destacando-se, mais uma vez, a importância do acompanhamento farmacoterapêutico, com resultados positivos à saúde do paciente?

Tabela 2 - Automedicação entre idosos do grupo da "Melhor Idade" da Faculdade de Ciências e Educação Sena Aires (FACESA) de Valparaíso do Goiás-GO ( $n=30)$.

\begin{tabular}{c|c|c} 
Ingestão de medicamentos & $\mathrm{n}$ & $\mathrm{f} \% \mathrm{p}$ \\
\hline Tomam medicamento por conta própria & 17 & 56,7 \\
\hline Não tomam medicamentos por conta própria & 13 & 43,3 \\
\hline Consomem chá ou garrafada & 19 & 63,3 \\
\hline Não consomem chá ou garrafada & 11 & 36,7 \\
\hline Alérgicos a algum medicamento & 03 & 10,0 \\
\hline Não alérgicos & 27 & 90,0 \\
\hline Legenda: $\mathrm{n}=$ número de idosos. f\%p = Frequência percentual
\end{tabular}

Ao analisar o uso de medicamentos, contatou-se interação medicamentosa em 43,3\% (n=13) dos idosos (Tabela 3). A presença do farmacêutico é minimizar ao máximo as interações medicamentosas com o objetivo de que o indivíduo tenha uma melhor qualidade de vida. AAtenção Farmacêutica no Brasil tem mostrado resultados significativos na diminuição dos problemas relacionados a medicamentos ${ }^{14}$.

Tabela 3 - Interação medicamentosa em idosos do grupo da "Melhor Idade" da Faculdade de Ciências e Educação Sena Aires (FACESA) de Valparaíso do Goiás-GO ( $\mathrm{n}=30)$.

\begin{tabular}{c|c|c} 
Interações medicamentosas & $\mathrm{n}$ & $\mathrm{f} \% \mathrm{p}$ \\
\hline Sem interações medicamentosas & 17 & 56,7 \\
\hline Com interações benéficas & 02 & 06,7 \\
\hline Com interações maléficas & 04 & 13,3 \\
\hline $\begin{array}{c}\text { Com automedicação, chás ou consumo de } \\
\text { álcool }\end{array}$ & 05 & 16,7 \\
\hline $\begin{array}{c}\text { Com associações de medicamentos no } \\
\text { decorrer do tempo }\end{array}$ & 02 & 06,7 \\
\hline
\end{tabular}

No Brasil, há uma grande prevalência de idosos que fazem uso de dois ou mais medicamentos ao dia, para isso 0 acompanhamento farmacoterapêutico surge para ter mais respostas positivas neste sentido?.

A presença do farmacêutico é de suma importância para auxiliar na melhoria da qualidade de vida do paciente, principalmente dos que fazem uso de vários tipos de medicamentos e que são mais vulneráveis as interações como os idosos. 


\section{Conclusão}

Observou-se, no presente estudo, maior prevalência de idosos que utiliza medicamento por conta própria e faz uso de chá ou garrafada, mesmo constituindo um dos grupos mais vulneráveis a intoxicação. Sendo assim, um acompanhamento farmacoterapêutico de qualidade é de suma importância, visto que é capaz de minimizar as interações medicamentosas dentre outros benefícios.

\section{Referências bibliográficas}

1. Chaimowicz F. A saúde dos idosos brasileiros às vésperas do século XXI: problemas, projeções e alternativas. Revista de Saúde Pública. 1997; 31(2): 184-200.

2. Foppa AA, Bevilacqua G, Pinto LH, Blatt CR. Atenção farmacêutica no contexto da estratégia de saúde da família. Revista Brasileira de Ciências Farmacêuticas. 2008; 44(4): 727-737.

3. Vieira FS. Possibilidades de contribuição do farmacêutico para a promoção da saúde. Ciência e saúde coletiva 2007; 12(1): 213-220.

4. Yokovama CS, Malucelli A, Moro CMC, Hirano LR, Nohama P. Proposta de sistema de informação para atenção farmacêutica baseado no Método Dáder. Revista de Ciências Farmacêuticas Básica e Aplicada 2011; 32(1) 19-26.

5. Holbach DM, Ferreira PM, Doblinski MF, Delaporte RH. Estudo do conhecimento dos profissionais farmacêuticos sobre a metodologia Dáder de atenção farmacêutica. Arq. Ciênc. Saúde Unipar 2006; 10(2): 81-86.

6. Comité de Consenso. Tercer Consenso de Granada sobre problemas relacionados con medicamentos (PRM) y resultados negativos asociados a la medicación (RNM). Ars Pharm. 2007; 48(1): 5-17.

7. Stuaro D. A importância do acompanhamento farmacoterapêutico em pacientes onco-hematológicos. Revista Brasileira de Hematologia e Hemoterapia 2009; 31(3):124.

8. Machuca M, Fernandez-llimos F, FAUS MJ. Metodo Dáder. Guia de Seguimento Farmacoterapêutico. 3rd ed. GIAFUGR; 2003.

9. Oliveira MPF, Novaes M. R. C. G. Drug-related problems in institutionalized elderly in Brasilia, Brazi. Biomedicine \& Aging Pathology 2011; 1: 179-184.

10. Lieber NSR, Teixeira JJV, Farhat FCLG, Ribeiro E, Crozzatti MTL, Oliveira GSA. Revisão dos estudos de intervenção do farmacêutico no uso de medicamentos por pacientes idosos. Cad. Saúde Pública 2002; 18(6): 1499-1507.

11. Silva FAB. Faculdade de Ciências e Educação Sena Aires. CPA - Comissão Própria de Avaliação. 2006-2008. Disponível em: <http://www.senaaires.com.br/facesa/CPA/ RELATORIO_FINAL_-_CPA_TRIENIO_2006-2008.pdf>. Acesso em: 30 set. 2012.

12. Amado LR, Carniel TZ, Restini CBA. Interações medicamentosas de anticoncepcionais com antimicrobianos e álcool relacionando à prática de automedicação. 2011; 13(7): 1451-1465.

13. Codogno JS, Fernandes RA, Monteiro HL. Prática de atividades físicas e custo do tratamento ambulatorial de diabéticos tipo 2 atendidos em unidade básica de saúde. 2012; 56(1): 6-11.

14. Pereira LRL, Freitas O. A evolução da Atenção Farmacêutica e a perspectiva para o Brasil. Revista Brasileira de Ciências Farmacêuticas. 2008; 44(4): 601-612.

\section{Nathana Lima de Sá}

Endereço para correspondência - RQE 17. CJ. M. casa 15. Bairro Guará 2, CEP 71050-132, Brasília, DF, Brasil.

E-mail: nathana.lima@gmail.com

Currículo Lattes: http://lattes.cnpq.br/2168923124214498

Renata Costa Fortes - fortes.rc@gmail.com

Recebido em 09 de setembro de 2013.

Publicado em 30 de janeiro de 2014. 